\title{
Preoperative high-dose methylprednisolone and glycaemic control early after total hip and knee arthroplasty - a RCT
}

Viktoria Lindberg-Larsen ${ }^{1,2}$, Henrik Kehlet ${ }^{1,2}$, Jens Bagger ${ }^{3}$, Sten Madsbad ${ }^{4}$

${ }^{1}$ Section for Surgical Pathophysiology 7621, Copenhagen University Hospital, Rigshospitalet, Denmark; ${ }^{2}$ The Lundbeck Foundation Centre for Fast-Track Hip and Knee Arthroplasty, Copenhagen, Denmark; ${ }^{3}$ Department of Orthopaedic Surgery, Copenhagen University Hospital, Bispebjerg and Frederiksberg, Denmark; ${ }^{4}$ Department of Endocrinology, Copenhagen University Hospital, Hvidovre, Denmark

\section{INTRODUCTION}

The surgical stress response includes inflammatory and endocrine-metabolic responses with subsequent changes in glucose metabolism leading to insulin resistance and hyperglycaemia.

Glucocorticoids are known to reduce the systemic inflammatory response after surgery. On the other hand, acute glucocorticoid therapy is associated with changes in glucose homeostasis; supressing insulin secretion, inducing hepatic insulin resistance promoting hepatic glucose production, and inhibiting glucose uptake. The aim of our RCT was to evaluate the effect of preoperative high-dose methylprednisolone (MP) $125 \mathrm{mg}$ iv. on glucose homeostasis after fast-track hip and knee arthroplasty.

\section{METHODS}

134 patients undergoing elective unilateral THA and TKA were randomized (1:1) receiving preoperative MP $125 \mathrm{mg}$ iv. (group MP) or isotonic saline iv. (group C). All procedures were performed under spinal anaesthesia without tourniquet and drains, using a standardized multimodal analgesic regime.

The primary outcome was change in plasma glucose concentrations between groups from baseline to 48 postoperatively. Secondary outcomes were change in plasma Cpeptide concentrations, homeostatic model assessment (HOMA) for insulin resistance (-IR) and $\beta$-cell function (-B).

Blood samples were collected at baseline (T0) and 2 (T2), 6 (T6), 24 (T24) and 48 (T48) hours after surgery, and complete blood samples from 122 patients were available for analyses (Figure 1).

Approvals: Ethics Committee (H-6-2014-101 and H-15007653) and the Danish Health and Medicine Authority (EudraCT 2014-003395-23 and 2015-000102-19).

ClinicalTrials.gov: NCT02332603

\section{RESULTS}

MP significantly increased plasma glucose at $\mathrm{T} 2 ; 7.4 \mathrm{mmol} \mathrm{l}^{-1}(95 \%$ CI 7.2-7.5) vs. $6.0 \mathrm{mmol} \mathrm{l}^{-1}$ (5.9-6.2), $\mathrm{p}=0.023$, and at T6 (non-fasting); $13.9 \mathrm{mmol} \mathrm{l}^{-1}$ (13.3-14.5) vs. $8.4 \mathrm{mmol} \mathrm{l}^{-1}(7.8-9.0)$, $\mathrm{p}<0.001$ (fig. $2 \mathrm{~A}$ ), as well as C-peptide at T24; $1675 \mathrm{pmol}$ $1^{-1}(1573-1778)$ vs. 1248 pmol l-1 $^{-1}(1145-1351), \mathrm{p}<0.001$ (fig. 2B).

Comparing non-fasting T6 glucose and C-peptide, group MP presented an impaired insulin response reflected by HOMA-B ( $<<0.001)$ (fig. 2D). Additionally, HOMAIR increased at T24 in group MP compared to group C ( $<<0.001)$ (fig. $2 \mathrm{C}$ ).

Parameters were normalized 48 hours postoperatively.

\section{DISCUSSION \& CONCLUSION}

Our study illustrates the acute but transient effect of high-dose glucocorticoid on glucose metabolism. Already two hours after surgery the glucose concentration was significantly higher in group MP compared with group $C$, indicating the inability of group MP to compensate adequately with increased insulin secretion in response to the glucocorticoid-induced insulin resistance. The effect of glucocorticoid on both $\beta$ cell function and insulin resistance was transient, and the effect of glucocorticoidinduced hyperglycaemia $\left(>10 \mathrm{mmol} \mathrm{l}^{-1}\right)$ for 1-2 days on perioperative morbidity in a fast-track setting is unknown and calls for further investigation.

Preoperative administration of high-dose MP resulted in transient disturbances in glucose homeostasis after elective THA and TKA.

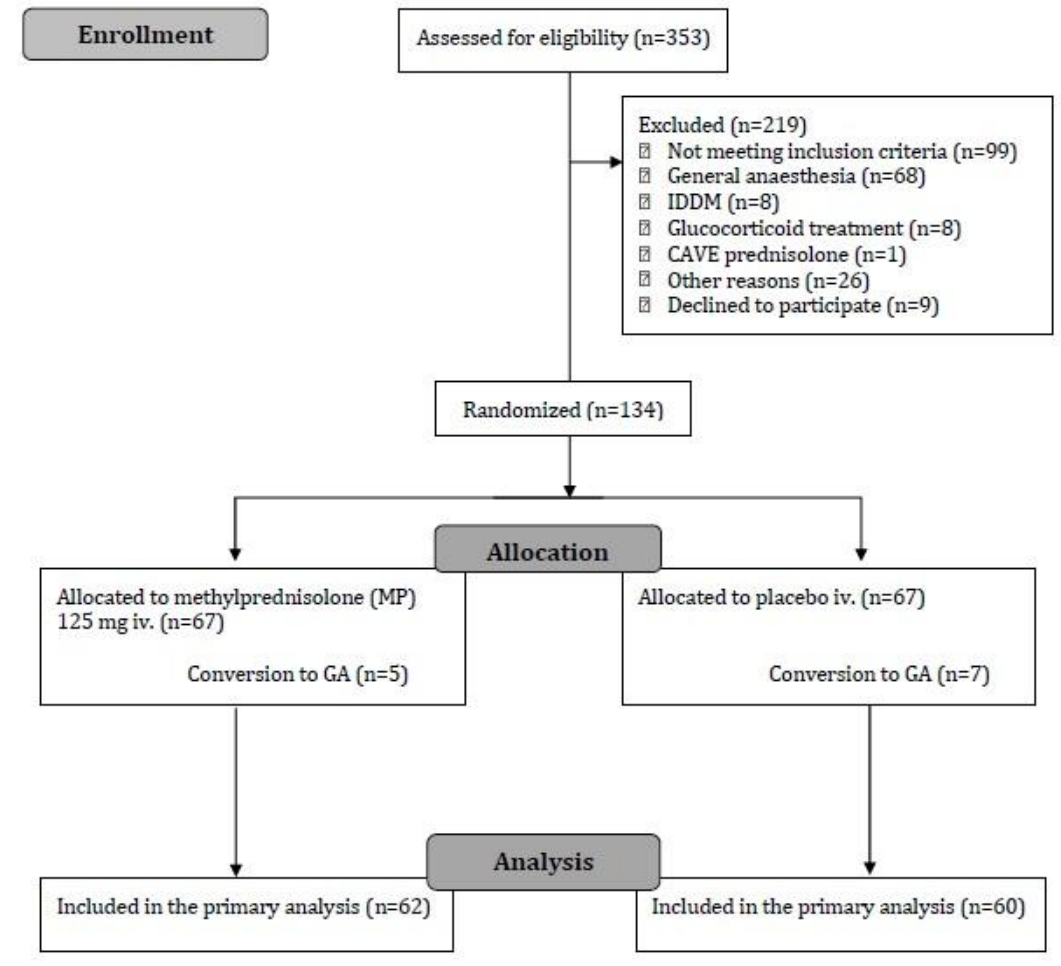

Figure 1.

CONSORT flow diagram for screening, inclusion, and exclusion of trial participants.

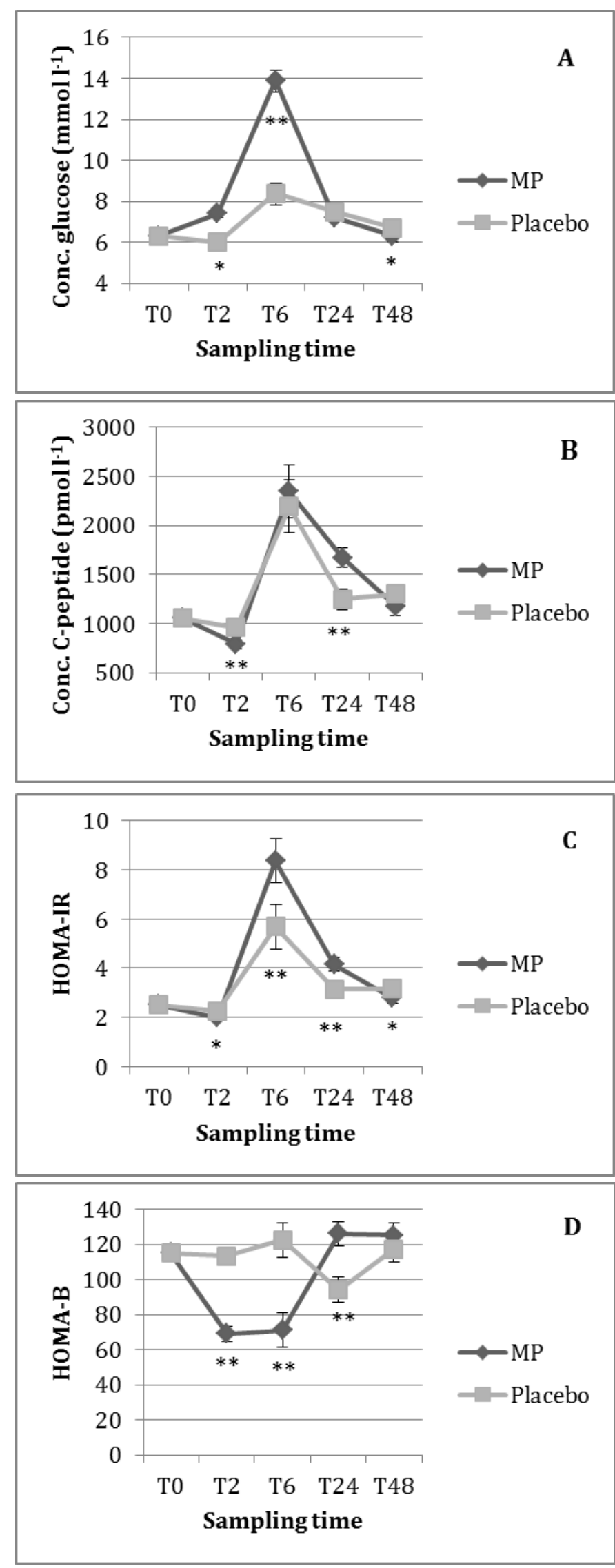

Figure 2.

Glycaemic markers. 